\title{
Optic chiasm measurements may be useful markers of anterior optic pathway degeneration in neuromyelitis optica spectrum disorders
}

\author{
Valentin Juenger ${ }^{1,2,3} \cdot$ Graham Cooper $^{1,2,4,5} \cdot$ Claudia Chien $^{1,2} \cdot$ Meera Chikermane $^{1,2} \cdot$ Frederike Cosima Oertel $^{1,2,6}$. \\ Hanna Zimmermann ${ }^{1,2} \cdot$ Klemens Ruprecht $^{7}$. Sven Jarius ${ }^{8} \cdot$ Nadja Siebert ${ }^{2}$ • Joseph Kuchling ${ }^{2,7}$. \\ Athina Papadopoulou ${ }^{2,9} \cdot$ Susanna Asseyer ${ }^{2,7}$. Judith Bellmann-Strobl ${ }^{1,2}$. Friedemann Paul ${ }^{1,2,4,7}$. \\ Alexander U. Brandt ${ }^{2,10}$. Michael Scheel ${ }^{2,3}$
}

Received: 14 January 2020 / Revised: 1 March 2020 / Accepted: 1 April 2020 / Published online: 26 April 2020

(C) The Author(s) 2020

\begin{abstract}
Objectives We aimed to evaluate optic chiasm (OC) measures as potential imaging marker for anterior optic pathway damage assessment in the context of neuromyelitis optica spectrum disorders (NMOSD).

Materials and method This cross-sectional study included 39 patients exclusively with aquaporin 4-IgG seropositive NMOSD of which 25 patients had a history of optic neuritis (NMOSD-ON) and 37 age- and sex-matched healthy controls (HC). OC heights, width, and area were measured using standard 3D T1-weighted MRI. Sensitivity of these measures to detect neurodegeneration in the anterior optic pathway was assessed in receiver operating characteristics analyses. Correlation coefficients were used to assess associations with structural measures of the anterior optic pathway (optic nerve dimensions, retinal ganglion cell loss) and clinical measures (visual function and disease duration).

Results OC heights and area were significantly smaller in NMOSD-ON compared to HC (NMOSD-ON vs. HC $p<0.0001)$. An OC area smaller than $22.5 \mathrm{~mm}^{2}$ yielded a sensitivity of 0.92 and a specificity of 0.92 in separating chiasms of NMOSD-ON from HC. OC area correlated well with structural and clinical measures in NMOSD-ON: optic nerve diameter $(r=0.4, p=0.047)$, peripapillary retinal nerve fiber layer thickness $(r=0.59, p=0.003)$, global visual acuity $(r=-0.57, p=0.013)$, and diseases duration $(r=-0.5, p=0.012)$.

Conclusion Our results suggest that OC measures are promising and easily accessible imaging markers for the assessment of anterior optic pathway damage.
\end{abstract}

Alexander U. Brandt and Michael Scheel contributed equally to this article.

Friedemann Paul

friedemann.paul@charite.de

1 Experimental and Clinical Research Center, Max Delbrueck Center for Molecular Medicine and Charité - Universitätsmedizin Berlin, corporate member of Freie Universität Berlin, Humboldt-Universität zu Berlin and Berlin Institute of Health, Berlin, Germany

2 NeuroCure Clinical Research Center, Charité - Universitätsmedizin Berlin, corporate member of Freie Universität Berlin,

Humboldt-Universität zu Berlin and Berlin Institute of Health, Charitéplatz 1, 10117 Berlin, Germany

3 Department of Neuroradiology, Charité - Universitätsmedizin Berlin, corporate member of Freie Universität Berlin, Humboldt-Universität zu Berlin and Berlin Institute of Health, Berlin, Germany

4 Einstein Center for Neurosciences, Berlin, Germany
5 Department of Experimental Neurology and Center for Stroke Research Berlin, Charité - Universitätsmedizin Berlin, Berlin, Germany

6 Multiple Sclerosis Center, Dept. of Neurology, University of California San Francisco, San Francisco, CA, USA

7 Department of Neurology, Charité - Universitätsmedizin Berlin, corporate member of Freie Universität Berlin, Humboldt-Universität zu Berlin and Berlin Institute of Health, Berlin, Germany

8 Molecular Neuroimmunology Group, Department of Neurology, University of Heidelberg, Heidelberg, Germany

9 Neurologic Clinic and Policlinic, Departments of Medicine, Clinical Research and Biomedicine University Hospital Basel,

Basel, Switzerland

10 Department of Neurology, University of California, Irvine, CA, USA 


\section{Key Points}

- Optic chiasm dimensions were smaller in neuromyelitis optica spectrum disorder patients compared to healthy controls.

- Optic chiasm dimensions are associated with retinal measures and visual dysfunction.

- The optic chiasm might be used as an easily accessible imaging marker of neurodegeneration in the anterior optic pathway with potential functional relevance.

Keywords Optic neuritis · Optic chiasm · Magnetic resonance imaging · Neuromyelitis optica

$\begin{array}{ll}\text { Abbreviations } & \\ \text { AQP4-IgG } & \text { Aquaporin 4-IgG } \\ \text { AUC } & \text { Area under the curve } \\ \text { CI } & 95 \% \text { confidence interval } \\ \text { CoV } & \text { Coefficient of variation } \\ \text { EDSS } & \text { Expanded Disability Status Scale } \\ \text { GCIPL } & \text { Combined ganglion cell-inner } \\ & \text { plexiform layer } \\ \text { HC } & \text { Healthy controls } \\ \text { ICC } & \text { Intraclass correlations } \\ \text { logMAR } & \text { Logarithm of the minimum } \\ & \text { angle of resolution } \\ \text { NMOSD } & \text { Neuromyelitis optica spectrum disorders } \\ \text { NMOSD-ON } & \text { Neuromyelitis optica patients with } \\ & \text { history of optic neuritis } \\ \text { NMOSD-NON } & \text { Neuromyelitis optica patients without } \\ \text { history of optic neuritis } & \text { Optic chiasm } \\ \text { OC } & \text { Optical coherence tomography } \\ \text { OCT } & \text { Optic neuritis } \\ \text { ON } & \text { Peripapillary retinal nerve fiber layer } \\ \text { pRNFL } & \text { Receiver operating characteristics } \\ \text { ROC } & \\ & \end{array}$

\section{Introduction}

Neuromyelitis optic spectrum disorders (NMOSDs) are inflammatory autoimmune CNS diseases that preferentially target the optic nerves and are frequently associated with serum autoantibodies to aquaporin-4 [1, 2]. Optic pathway degeneration following optic neuritis $(\mathrm{ON})[3,4]$ results in atrophy involving the entire visual pathway [5-10]. At the optic chiasma (OC), fibers from the left and the right optic nerve merge and form the site of highest axonal density. Direct damage or other pathophysiological effects affecting the optic nerves may accumulate in the $\mathrm{OC}$, making it a promising target for the assessment of anterior optic pathway damage.

Measures of optic pathway degeneration are an important outcome measure of $\mathrm{ON}$, since they are related to impaired visual function and reduction of vision-related quality of life [11-14]. Optic pathway dimensions as assessed by MRI have been used as a surrogate marker of inflammatory damage and atrophy of the optic nerve and anatomically connected tracts $[3,4,15-18]$. It has been suggested that MRI is sensitive to axonal loss as a cause of optic nerve atrophy [17-20]. Optical coherence tomography (OCT) reveals damage to the retinal axons and ganglion cells by means of measuring peripapillary retinal nerve fiber layer ( $\mathrm{pRNFL}$ ) thickness and combined ganglion cell-inner plexiform layer (GCIPL) volume. OCT measures have been successfully used as surrogate markers of optic nerve atrophy $[16,17,19,21-25]$, being associated to MRI-detected macro- and microstructural optic pathway atrophy and visual function [16, 17, 20, 26-28].

Although MRI is used as part of the routine clinical workup of NMOSD patients [29, 30], no method to evaluate optic pathway damage has been established. In addition to the accumulation of damage within the anterior optic pathway, the OC appears particularly promising as a potential imaging marker, since it would simplify evaluation by reducing the region of interest from multiple structures to one. Only few studies have focused on the assessment of physiologic OC dimensions [31-33] and their changes in optic atrophy [18], while quantitative correlation analysis to visual function and optic pathway degeneration has not been performed.

The aim of this study was to assess whether neurodegenerative changes in the anterior optic pathway are detectable by assessing OC measures. We hypothesized that the OC assessment in standard 3D-T1w images is sensitive to anterior optic pathway damage. To test this hypothesis, we used NMOSD as a model for optic pathway damage and compared different OC measures (area, width, left, central, right, and total height) between aquaporin 4-IgG (AQP4-IgG) seropositive NMOSD patients with and without history of $\mathrm{ON}$ (NMOSD-ON and NMOSD-NON) and healthy controls (HC). In addition, we investigated the association of OC measures with optic nerve diameter, visual acuity, pRNFL thickness, and GCIPL volume in NMOSD-ON.

\section{Material and methods}

\section{Study population}

Data of 78 NMOSD patients acquired from an ongoing longitudinal prospective observational cohort study at the NeuroCure Clinical Research Center, CharitéUniversitätsmedizin Berlin (recruited from May 2013 to January 2018) were screened for eligibility. All patients (i) 
were 18 years or older and (ii) had a diagnosis of AQP4-IgG seropositive NMOSD according to the current panel criteria [29] and (iii) either had a last ON attack at least 5 months prior to MRI or had no history of ON. AQP4-IgG status was determined by a cell-based assay (Euroimmun, Lübeck, Germany). Patients with AQP4-IgG seronegative $(n=25)$ antibody status, unknown antibody status and/or incomplete clinical data $(n=10)$, lacking MRI data $(n=3)$, or ON within 5 months prior to MRI $(n=1)$ were excluded.

Thirty-nine patients exclusively with AQP4-IgG seropositive NMOSD and 37 age- and sex-matched HC subjects were included in this study (Table 1). All HC subjects were 18 years or older and had ophthalmologic testing and no history of neurological or ophthalmological diseases.

This study was approved by the local ethics committee (Ethikkommission der Charité-Universitätsmedizin Berlin; EA1/131/09) and conducted according the declaration of Helsinki and applicable German law. All participants gave written informed consent.

\section{MRI acquisition}

MRI data were acquired on the same 3-T scanner (MAGNETOM Trio, A Tim System, Siemens) at the Berlin Center for Advanced Neuroimaging using a volumetric highresolution T1-weighted MPRAGE sequence $(\mathrm{RT}=1900 \mathrm{~ms}$, $\mathrm{TE}=3.03 \mathrm{~ms}, \mathrm{TI}=900 \mathrm{~ms}, \mathrm{FOV}=256 \times 256 \mathrm{~mm}^{2}$, matrix $256 \times 256$, slice thickness $1 \mathrm{~mm}$ ). OC measurements were performed on reconstructed 3D MPRAGE MR images.

\section{Optic chiasm measures}

After training with a neuroradiologist with more than 8 years of experience (M.S.), OC and optic nerve measurements were performed by V.J. (radiology trainee), blinded to clinical data, using a standardized protocol: First, the central point of the OC was determined on all 3 planes. The axes of the planes were reoriented to the course of the optic pathway, so that they were perpendicular to the orientation of the individual $\mathrm{OC}$ in the central point. On the individually reoriented transversal plane, OC area, heights, and width were measured. Optic nerve diameters were measured in the cisternal segment $7 \mathrm{~mm}$ anterior to the previously defined central point of the OC perpendicular to the optic nerve course (Figs. 1 and 2). All measurements were performed using region-of-interest software from Horos, version 3.3.2 (https:// www.horosproject.org). Width was defined as the diameter along the adjusted frontal axis. Heights were measured perpendicularly to that diameter: central height at the middle, the lateral heights at the maximal diameter left and right to the center. For interrater reliability analysis, J.V. and M.C. (trainee) measured OC dimensions within 10 randomly selected $\mathrm{HC}$ and intraclass correlations (ICC) were calculated.

\section{Clinical assessment}

Neurological disability was on the Expanded Disability Status Scale (EDSS), including the visual functional system score according to the Neurostatus definitions [34]. Raters were

Table 1 Demographics and clinical characteristics

\begin{tabular}{|c|c|c|c|c|}
\hline & $\mathrm{HC}$ & $\begin{array}{l}\text { NMOSD- } \\
\text { NON }\end{array}$ & NMOSD-ON & $p$ \\
\hline Number & 37 & 14 & 25 & - \\
\hline Age, years (mean, SD) & $47.8(12.6)$ & $53.8(12.5)$ & $48.09(14.9)$ & 0.33 \\
\hline $\operatorname{Sex}(\mathrm{F} / \mathrm{M})(\%$ female $)$ & $30 / 7(81 \%)$ & $14 / 0(100 \%)$ & $21 / 4(84 \%)$ & 0.22 \\
\hline Disease duration, years (median, range) & - & - & $7.1(3-34.1)$ & \\
\hline Number of ON (median, range) & - & - & $2(1-12)$ & - \\
\hline Time since first ON, years (median, range) & - & - & $6.9(3.4-32.2)$ & - \\
\hline Time since last ON, years (median, range) & - & - & $4.9(0.5-13.2)$ & - \\
\hline ON involvement, bilateral/unilateral & - & - & $\begin{array}{l}11 / 14 \\
(8 \text { right } / 6 \text { left })\end{array}$ & - \\
\hline Optic nerve diameter, mm (mean, SD) & $8.37(0.50)$ & $8.13(0.90)$ & $7.06(1.23)$ & $<0.001$ \\
\hline pRNFL, $\mu \mathrm{m}$ (mean, SD) & $91.99(15.10)$ & $97.96(10.13)$ & $67.57(17.92)$ & $<0.001$ \\
\hline GCIPL mm³ (mean, SD) & $1.83(0.28)$ & $1.88(0.14)$ & $1.49(0.25)$ & $<0.001$ \\
\hline Visual acuity, logMAR (mean, SD) & $-0.01(0.21)$ & $-0.15(0.21)$ & $0.30(0.63)$ & 0.04 \\
\hline Visual Functional System Score (median, range) & - & $0(0-2)$ & $1(0-6)$ & $<0.001$ \\
\hline EDSS (median, range) & - & $3(1-7)$ & $4(0-6.5)$ & 0.71 \\
\hline
\end{tabular}

$p$ values are groupwise comparisons. $H C=$ healthy controls; $N M O S D-O N=$ neuromyelitis optica patients with history of optic neuritis; $N M O S D-N O N=$ neuromyelitis optica patients without history of optic neuritis; $O N=$ optic neuritis; $p R N F L=$ peripapillary retinal nerve fiber layer; $G C I P L=$ combined ganglion cell-inner plexiform layer; $\log M A R=\operatorname{logarithm}$ of the minimum angle of resolution; $E D S S=$ Expanded Disability Status Scale 


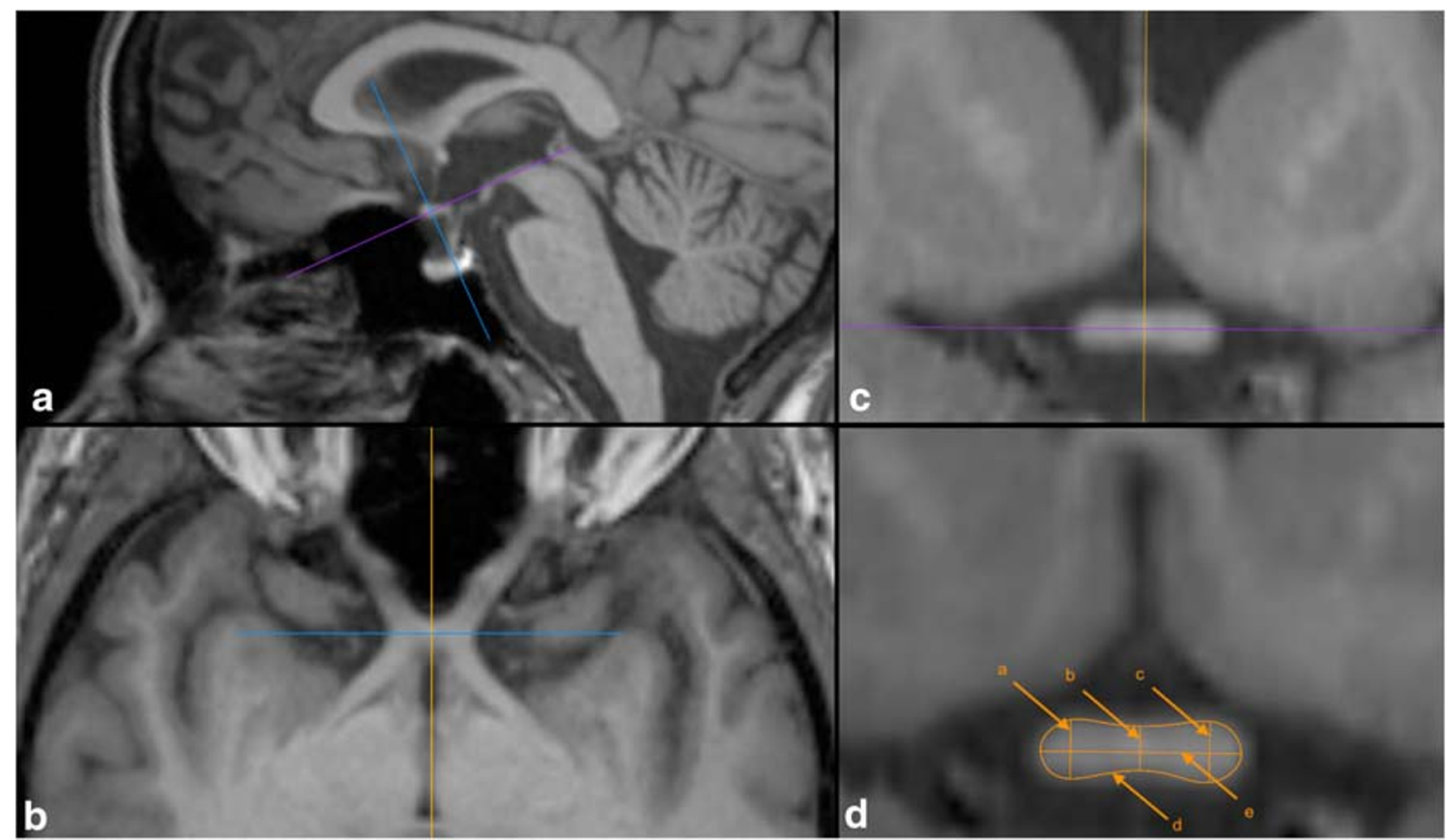

Fig. 1 Optic chiasm measurement. Panels $\mathbf{a}, \mathbf{b}$, and $\mathbf{c}$ illustrate how axes were adjusted perpendicularly to the orientation of the OC in the center point. Panel d shows a sample OC measurement ( $\mathrm{a}=$ right, $\mathrm{b}=$ central, $\mathrm{c}=$ left height, $\mathrm{d}=$ area, $\mathrm{e}=$ width)

under the supervision of board-certified neurologists. The global neurological examination also included assessment of ON history using clinical criteria. Visual acuity was tested monocularly under phototopic conditions using retroilluminated Early Treatment in Diabetes Retinopathy Study charts at a 4-m distance. The logarithm of the minimum angle of resolution (logMAR) served as a measure of visual function. Visual acuity data was included only from patients where best correction was used $(n=30)$.

\section{Optical coherence tomography measures}

All OCT data were acquired on a spectral domain OCT device (Spectralis, Heidelberg Engineering) with automated real-time function. No pupil dilatation was used. We report the OCT acquisition settings and scanning protocol according to the APOSTEL recommendations [35]: The pRNFL thickness was measured using 3.4-mm ring scans around the optic nerve head $\left(12^{\circ}, 1536 \mathrm{~A}\right.$-scans, $\left.9 \leq \mathrm{ART} \leq 100\right)$. The GCIPL volume was measured using a 6-mm diameter cylinder around the fovea from a macular volume scan $\left(25^{\circ} \times 30^{\circ}, 61\right.$ vertical
B-scans, 768 A-scans per B-scan, ART = 15). Segmentation of the pRNFL and the intraretinal layers in the macular scan was performed semi-automatically using software provided by the optical coherence tomography manufacturer (Eye Explorer 1.9.10.0 with viewing module 6.0.9.0; Heidelberg Engineering). Quality was evaluated according to the OSCAR-IB criteria [36, 37].

Two patients did not have OCT data. Eight eyes from six NMOSD-ON had to be excluded due to incidental findings or quality reasons. Only the macular scan from two additional NMOSD-ON eyes was excluded due to quality reasons.

\section{Statistics}

Proportional group differences were tested with $\chi^{2}$ test for sex and with ANOVA test for age. For comparison of ordinal and continuous measurements, groupwise comparison was performed using Kruskal-Wallis and ANOVA tests, respectively. Group comparison of OC dimensions was corrected for multiple comparison using the Holm-Bonferroni method. The variations of the individual metrics were compared within the $\mathrm{HC}$

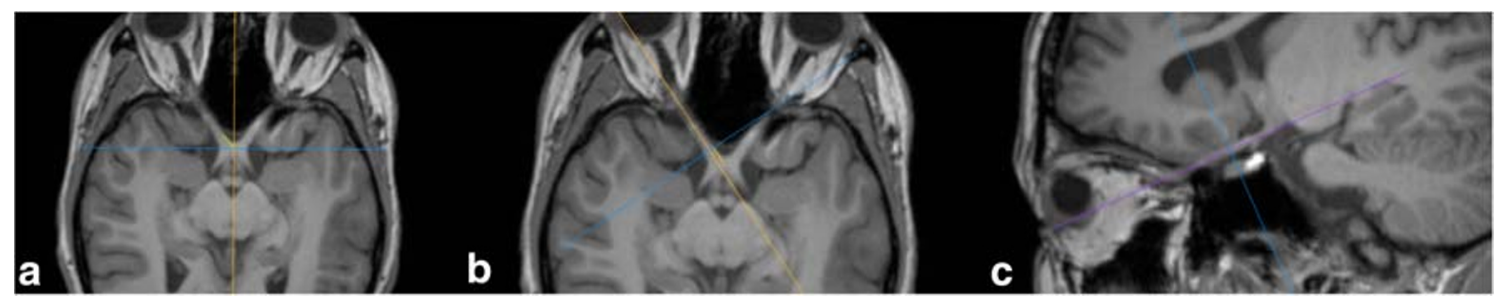

Fig. 2 Optic nerve measurement. Panels $\mathbf{a}, \mathbf{b}$, and $\mathbf{c}$ illustrate how axes were adjusted perpendicularly to the orientation of the optic nerve 
group using a coefficient of variation $(\mathrm{CoV})$ computed according to

$\mathrm{CoV}=\frac{\text { standard deviation of the individual metric }}{\text { mean of the individual metric }}$

and served as an indicator of variation in the individual OC measures. Sensitivity to optic nerve atrophy of individual OC measures was evaluated with receiver operating characteristic (ROC) analysis, including area under the curve (AUC) comparison using DeLong method [38]. Association analysis of individual OC measures with the T2 lesion load, the SIENAX V-scaling [39] factor for head size and gender as potential influencing factors, mean optic nerve diameter, mean pRNFL thickness, mean GCIPL volume, visual function (mean $\log \mathrm{MAR}$ ), and disease duration was performed with the Pearson correlation test, association with the number of ON attacks with the Spearman test.

Statistical analyses were performed using R software, version 3.5.1. (http://www.r-project.org/) with the tidyverse [40], ggpubr [41], and pROC packages [42]. Statistical significance was set at a $p$ value $<0.05$.

\section{Results}

\section{Demographics}

Table 1 shows the demographic and clinical characteristics of the cohort. No significant differences of sex distribution, age, and physical disability were found between groups. Optic nerve diameters were different in NMOSD-ON compared to $\mathrm{HC}(p<0.0001)$, NMOSD-ON compared to NMOSD-NON $(p<0.01)$, but not in NMOSD-NON compared to $\mathrm{HC}$ $(p>0.05)$.

\section{Group comparison and receiver operating characteristics}

Figure 3 shows the OC of NMOSD-ON, NMOSD-NON, and $\mathrm{HC}$ to illustrate the reduction of OC dimensions in NMOSD.

All OC measures except width were significantly smaller in all group comparisons (NMOSD-ON vs. HC: $p<0.0001$; NMOSD-NON vs. HC: $p<0.01$; and NMO-ON vs. NMONON: $p<0.03$ ), as shown in Table 2 and Fig. 4 . When correcting for multiple comparisons (corrected $p=0.003$ ), this remained significant for all measures for HC vs. NMOSD$\mathrm{ON}$, for all measures except left height for HC vs. NMOSDNON and for area for NMOSD-NON vs. NMOSD-ON.

OC measures were not associated with the T2 lesion load $(r=-0.35, p>0.09)$, the number of ON attacks $(r=-0.31$, $p>0.13)$, the number of ON attacks per side $(r=-0.09$, $p>0.13)$, the SIENAX V-scaling factor, or gender $(r<0.08$, $p>0.23)$. Thus, no correction for head size or sex was performed.

A ROC analysis was conducted to test the ability of the OC to predict the presence of damage in the anterior optic pathway, namely to differentiate between groups. OC area and $\mathrm{OC}$ heights have comparable AUC values for each group (NMOSD-ON vs. HC: AUC > 0.92; NMOSD-NON vs. HC: AUC > 0.74; NMO-ON vs. NMO-NON: $\mathrm{AUC}>0.71$ ), whereas width has lower AUC values, as shown in Table 3 and Fig. 5. AUC comparison using DeLong method and variation comparison using the $\mathrm{CoV}$ of the best performing measures revealed no significant difference. An OC area smaller than $22.5 \mathrm{~mm}^{2}$ yielded a sensitivity of 0.92 and a specificity of 0.92 in separating chiasms of NMOSD-ON from HC.

\section{Associations with structural and clinical measures}

Table 4 summarizes the association analysis within the NMOSD-ON group. Higher OC measures were associated with bigger optic nerve diameter, better visual acuity, and better OCT measures. This was most prominent for OC area: Higher values significantly correlated with bigger optic nerve diameter $(r=0.4, p=0.047)$, better logMAR $(r=-0.57$, $p=0.013)$, thicker pRNFL $(r=0.59, p=0.003)$, bigger GCIPL $(r=0.55, p=0.007)$, and shorter disease duration $(r=-0.5, p=0.012)$. Within OC heights, only central height was significantly associated with GCIPL $(r=0.46, p=0.028)$.

\section{Discussion}

We evaluated OC measures as imaging marker of anterior optic pathway damage. We demonstrated significant group differences between NMOSD patients and HC and strong associations of OC measures with structural and clinical measures. Our data show that OC assessment in standard 3D-T1w images is sensitive to anterior optic pathway damage. Hence, OC measures are easily accessible and sensitive markers of anterior optic pathway damage.

$\mathrm{OC}$ dimension values for $\mathrm{HC}$ presented in our study are similar to recently published data [33]. A previous study by Wagner et al [31], however, reported slightly higher OC width and area values. This study excluded OC height due to a high degree of variance. Notably, unlike our study, Wagner et al did not account for the transverse course of the optic pathway and did not define precise measure locations. Thus, the coronal plane would be at varying angles (not perpendicular) to the course of the optic pathway at which OC assessment results in higher values and variance. Furthermore, our study employed a higher resolution MRI sequence, which may have an impact on values and variance. 


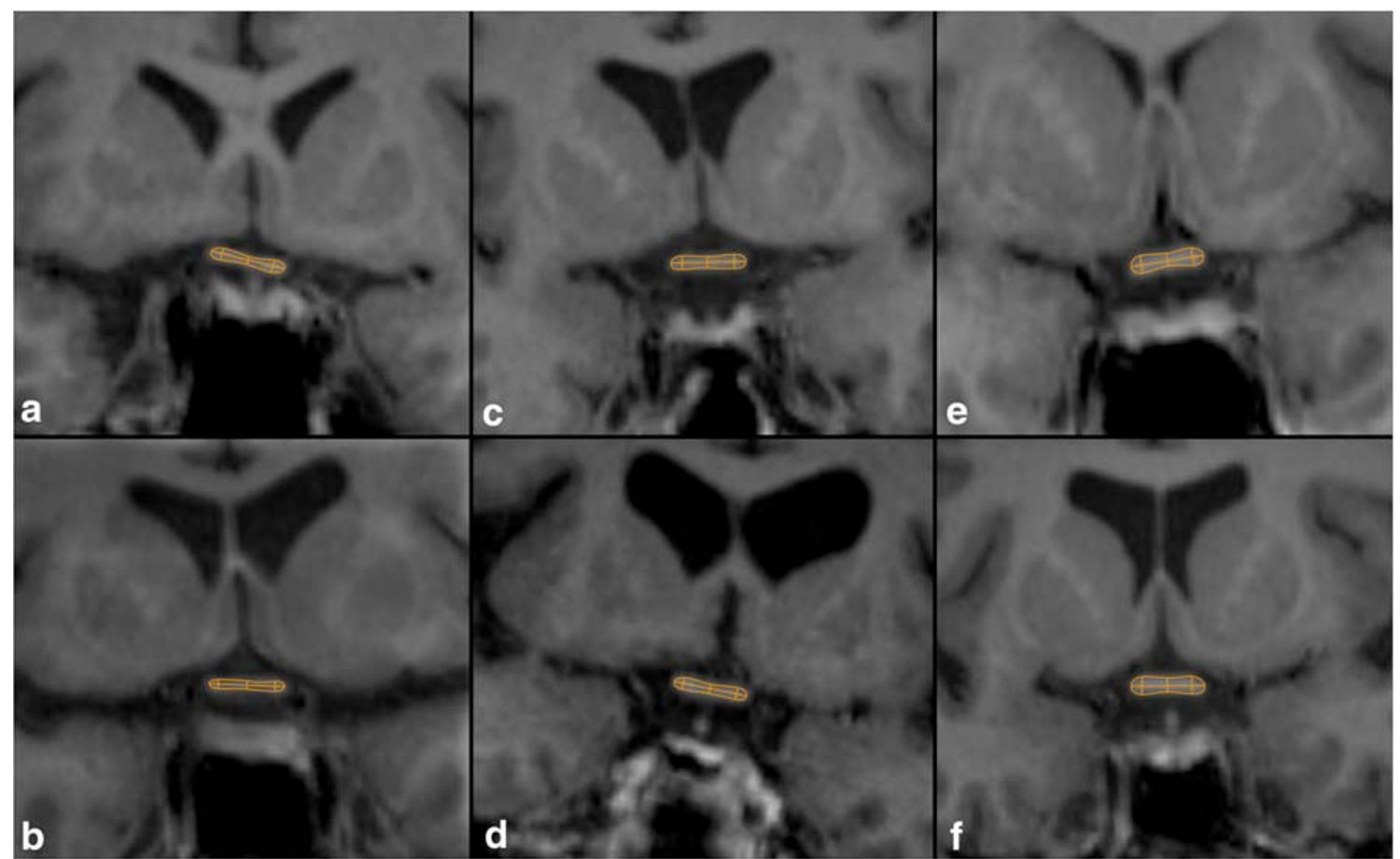

Fig. 3 Difference in optic chiasm dimensions. Shown are OCs of NMOSD-ON (a, b), NMOSD-NON (c, d), and HC (e, f)

OC heights and area showed differences between all groups. This is in line with earlier investigations suggesting that optic nerve dimensions discriminate ON patients from controls [16]. OC assessment, as suggested in our study, only requires a standard and broadly available MRI sequence (3D T1-weighted MPRAGE) and assesses a rather fixed structure less vulnerable to motion artifacts compared to the previously used orbital optic nerves $[3,4,16,17]$. Note that in this study optic nerve diameters were measured in the cisternal segment $7 \mathrm{~mm}$ anterior to the $\mathrm{OC}$, since contrast heterogeneity and motion artifacts in the orbital part rendered orbital assessment difficult in $25 \%$ of the patients. The observation that smaller OC dimensions were also found in NMOSD-NON (compared to $\mathrm{HC}$ and NMOSD-ON) supports microstructural changes in the optic pathway independent of ON [26], which have been described in NMOSD [5, 27, 43, 44]. In concordance with a study by Harrigan et al [16], the optic nerve diameter was smaller in patients with a history of ON compared to HC but not in patients without ON. Although this should not be overstated in consideration of the small NMOSD-NON sample size, this might indicate that microstructural changes independent of $\mathrm{ON}$, including anterograde degeneration

Table 2 Optic chiasm measures

\begin{tabular}{|c|c|c|c|c|c|c|c|c|}
\hline Measure & $\mathrm{HC}$ & $\begin{array}{l}\text { NMOSD- } \\
\text { NON }\end{array}$ & $\begin{array}{l}\text { NMOSD- } \\
\text { ON }\end{array}$ & $\begin{array}{l}\text { HC vs. NMOSD- } \\
\text { NON }\end{array}$ & $\begin{array}{l}\text { HC vs. NMOSD- } \\
\text { ON }\end{array}$ & $\begin{array}{l}\text { NMOSD-NON vs. NMOSD- } \\
\text { ON }\end{array}$ & $\mathrm{CoV}$ & ICC \\
\hline Left height (CI, mm) & $\begin{array}{l}2.77 \\
(0.35)\end{array}$ & $\begin{array}{l}2.34 \\
(0.53)\end{array}$ & $\begin{array}{l}1.94 \\
(0.48)\end{array}$ & $\begin{array}{l}t=2.77 \\
p=0.01\end{array}$ & $\begin{array}{l}t=7.49 \\
p<0.0001\end{array}$ & $\begin{array}{l}t=2.36 \\
p=0.03\end{array}$ & 0.12 & 0.71 \\
\hline $\begin{array}{l}\text { Central height (CI, } \\
\mathrm{mm})\end{array}$ & $\begin{array}{l}1.93 \\
(0.32)\end{array}$ & $\begin{array}{l}1.55 \\
(0.39)\end{array}$ & $\begin{array}{l}1.22 \\
(0.32)\end{array}$ & $\begin{array}{l}t=3.27 \\
p<0.001\end{array}$ & $\begin{array}{l}t=8.63 \\
p<0.0001\end{array}$ & $\begin{array}{l}t=2.70 \\
p=0.013\end{array}$ & 0.16 & 0.51 \\
\hline Right height (CI, mm) & $\begin{array}{l}2.65 \\
(0.36)\end{array}$ & $\begin{array}{l}2.20 \\
(0.46)\end{array}$ & $\begin{array}{l}1.79 \\
(0.43)\end{array}$ & $\begin{array}{l}t=3.31 \\
p=0.003\end{array}$ & $\begin{array}{l}t=8.23 \\
p<0.0001\end{array}$ & $\begin{array}{l}t=2.79 \\
p=0.008\end{array}$ & 0.14 & 0.77 \\
\hline Width (CI, mm) & $\begin{array}{l}12.23 \\
(1.15)\end{array}$ & $\begin{array}{l}12.17 \\
(1.05)\end{array}$ & $\begin{array}{l}11.43 \\
(1.87)\end{array}$ & $\begin{array}{l}t=0.17 \\
p=0.56\end{array}$ & $\begin{array}{l}t=1.91 \\
p=0.059\end{array}$ & $\begin{array}{l}t=1.59 \\
p=0.18\end{array}$ & 0.09 & 0.95 \\
\hline Area $\left(\mathrm{CI}, \mathrm{mm}^{2}\right)$ & $\begin{array}{l}27.07 \\
(3.50)\end{array}$ & $\begin{array}{l}22.26 \\
(4.65)\end{array}$ & $\begin{array}{l}16.89 \\
(4.44)\end{array}$ & $\begin{array}{l}t=3.51 \\
p=0.003\end{array}$ & $\begin{array}{l}t=9.61 \\
p<0.0001\end{array}$ & $\begin{array}{l}t=3.51 \\
p=0.001\end{array}$ & 0.13 & 0.89 \\
\hline Total height (CI, mm) & $\begin{array}{l}7.35 \\
(0.90)\end{array}$ & $\begin{array}{l}6.09 \\
(1.33)\end{array}$ & $\begin{array}{l}4.94 \\
(1.14)\end{array}$ & $\begin{array}{l}t=3.27 \\
p=0.003\end{array}$ & $\begin{array}{l}t=8.86 \\
p<0.0001\end{array}$ & $\begin{array}{l}t=2.73 \\
p=0.009\end{array}$ & 0.12 & 0.76 \\
\hline
\end{tabular}

Shown are means of OC measures. $p$ and $t$-values are derived from $t$ tests. Corrected $p=0.003$. $C I=95 \%$ confidence interval; $H C=$ healthy controls; $N M O S D-O N=$ neuromyelitis optica patients with history of optic neuritis; $N M O S D-N O N=$ neuromyelitis optica patients without history of optic neuritis; $C o V=$ coefficient of variation; $I C C=$ intraclass correlations 

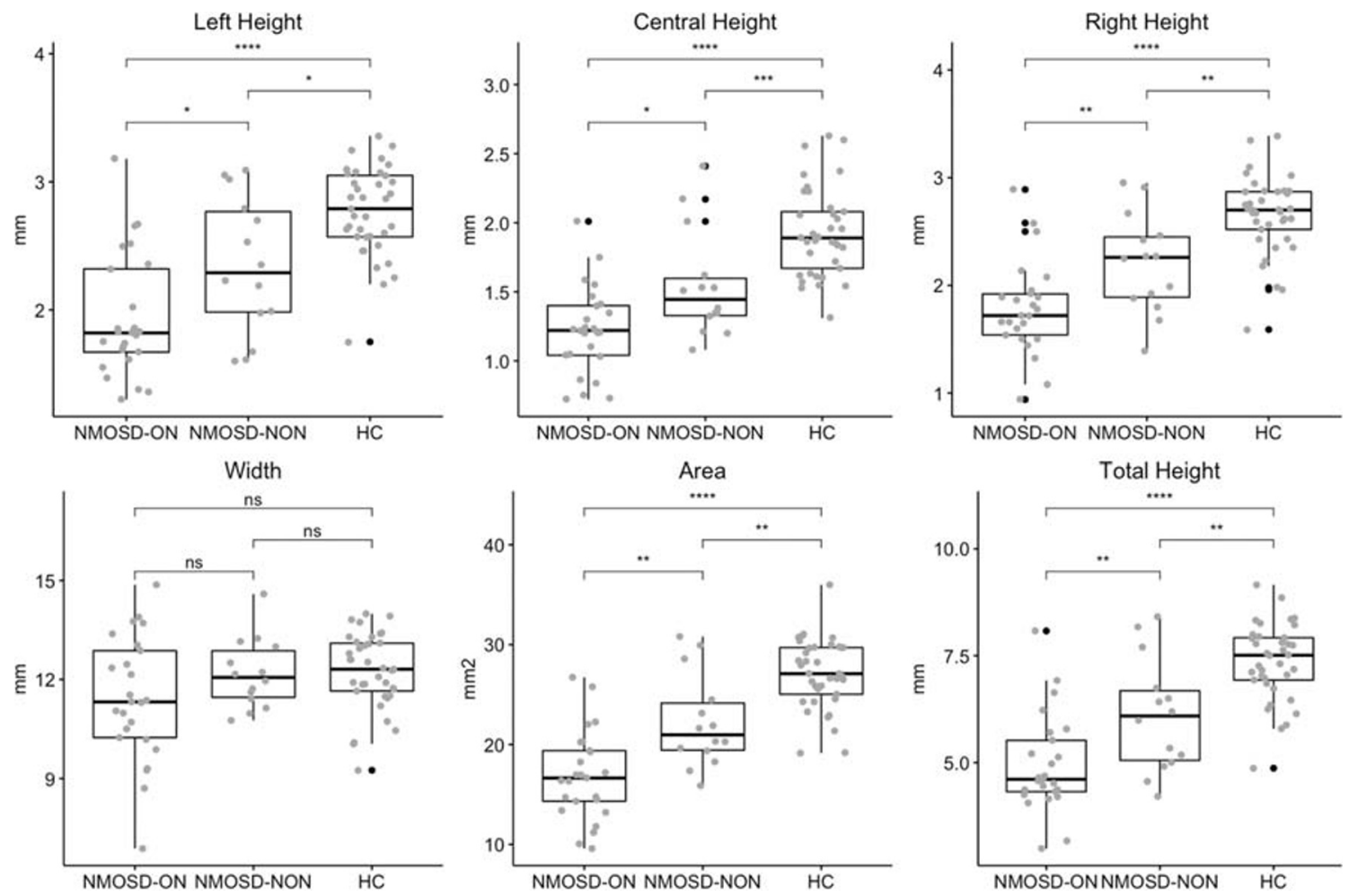

Fig. 4 Optic chiasm measures. Group differences in optic chiasm heights, width, and area between neuromyelitis optica spectrum disorders patients with optic neuritis (NMOSD-ON), without optic neuritis (NMOSD-

originating from anterior optic pathway damage but also retrograde degeneration originating from posterior optic pathway damage, might accumulate in the OC.

The AUC values obtained from ROC analyses for OC area and heights indicate that $\mathrm{OC}$ measures are sensitive to anterior
NON), and healthy controls (HC). ns: $p>0.05, * p \leq 0.05, * * p \leq 0.01$, $* * * p \leq 0.001, * * * * p \leq 0.0001$

optic pathway damage. On the descriptive level, OC left height shows slightly poorer ROC performance than right. This might rather be caused by an asymmetric severity of atrophy predominantly affecting fibers contributing to the right side of the OC (8 ON right vs. $6 \mathrm{ON}$ left), of handedness

Table 3 Receiver operating characteristics analysis

\begin{tabular}{|c|c|c|c|c|c|c|}
\hline HC vs. NMOSD-ON & Area & Width & $\begin{array}{l}\text { Left } \\
\text { Height }\end{array}$ & $\begin{array}{l}\text { Central } \\
\text { Height }\end{array}$ & $\begin{array}{l}\text { Right } \\
\text { Height }\end{array}$ & $\begin{array}{l}\text { Total } \\
\text { Height }\end{array}$ \\
\hline AUC & 0.95 & 0.64 & 0.90 & 0.95 & 0.92 & 0.94 \\
\hline $95 \% \mathrm{CI}$ & $0.91-1.00$ & $0.49-0.79$ & $0.81-0.98$ & $0.89-1.00$ & $0.85-1.00$ & $0.87-1.00$ \\
\hline$p$ value AUC comparison & - & $<0.01$ & 0.02 & 0.78 & 0.16 & 0.40 \\
\hline HC vs. NMOSD-NON & Area & Width & $\begin{array}{l}\text { Left } \\
\text { Height }\end{array}$ & $\begin{array}{l}\text { Central } \\
\text { Height }\end{array}$ & $\begin{array}{l}\text { Right } \\
\text { Height }\end{array}$ & $\begin{array}{l}\text { Total } \\
\text { Height }\end{array}$ \\
\hline AUC & 0.78 & 0.55 & 0.74 & 0.80 & 0.78 & 0.77 \\
\hline $95 \% \mathrm{CI}$ & $0.57-0.92$ & $0.38-0.73$ & $0.52-0.88$ & $0.59-0.95$ & $0.62-0.94$ & $0.60-0.94$ \\
\hline$p$ value AUC comparison & - & 0.08 & 0.31 & 0.49 & 0.93 & 0.77 \\
\hline NMOSD-ON vs. NMOSD-NON & Area & Width & $\begin{array}{l}\text { Left } \\
\text { Height }\end{array}$ & $\begin{array}{l}\text { Central } \\
\text { Height }\end{array}$ & $\begin{array}{l}\text { Right } \\
\text { Height }\end{array}$ & $\begin{array}{l}\text { Total } \\
\text { Height }\end{array}$ \\
\hline AUC & 0.81 & 0.63 & 0.71 & 0.74 & 0.76 & 0.76 \\
\hline $95 \% \mathrm{CI}$ & $0.69-0.95$ & $0.46-0.80$ & $0.54-0.89$ & $0.59-0.90$ & $0.57-0.90$ & $0.60-0.92$ \\
\hline$p$ value AUC comparison & - & 0.08 & 0.06 & 0.19 & 0.29 & 0.14 \\
\hline
\end{tabular}

Shown are area under the curve (AUC), $95 \%$ confidence interval (CI), and $p$ value for AUC comparison using area as reference. $H C=$ healthy controls; $N M O S D-O N=$ neuromyelitis optica patients with history of optic neuritis; NMOSD-NON= neuromyelitis optica patients without history of optic neuritis 

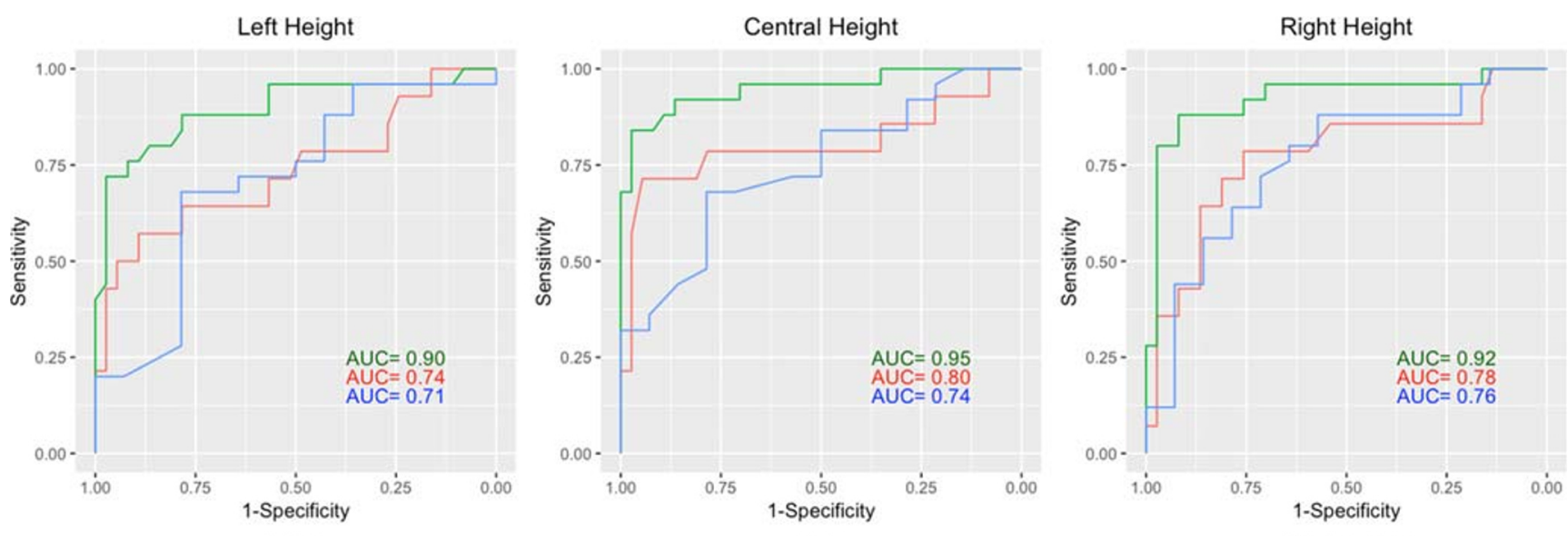

Width
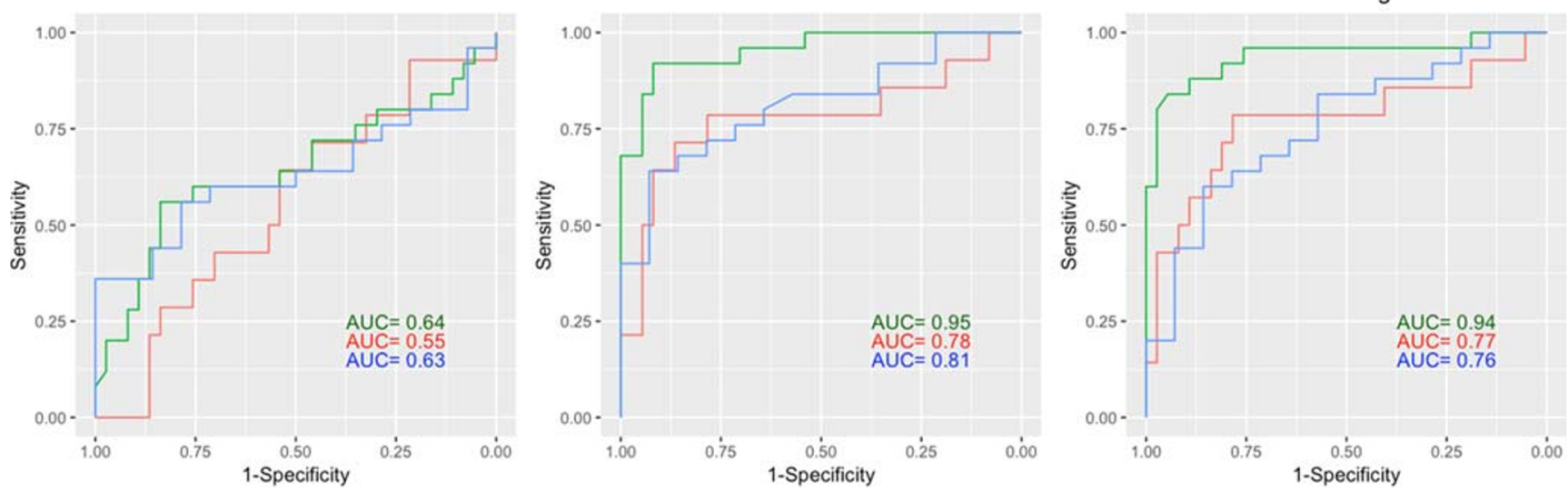

Fig. 5 Receiver operating characteristic for optic chiasm measures. Each line represents one group differentiation: green lines HC vs. NMOSD-ON, red lines $\mathrm{HC}$ vs. NMOSD-NON, blue lines NMOSD-ON vs. NMOSD-NON; ON = optic neuritis

or other asymmetries present in our study, than by a different sensitivity of the individual measure. This and the observation that no association between $\mathrm{ON}$ attacks per side and $\mathrm{OC}$ heights was found highlight the fact that OC assessment accumulates pathophysiologic processes of both sides and does not provide information on the origin of the fibers.
The observed associations between higher OC area and better visual acuity $(r=-0.57)$, thicker pRNFL $(r=0.53)$ and bigger GCIPL $(r=0.55)$ in NMOSD are similar to the associations reported on optic nerve dimensions $(r=-0.50$, $r=0.66, r=0.59)$ in MS [17]. The degree of association between $\mathrm{pRNFL}$ and anterior optic pathway dimensions in MRI
Table 4 Associations of OC measures and visual acuity, optic nerve diameter and optical coherence tomography measures for NMOSD-ON patients

\begin{tabular}{lllllll}
\hline Mean & Area & Width & $\begin{array}{l}\text { Left } \\
\text { Height }\end{array}$ & $\begin{array}{l}\text { Central } \\
\text { Height }\end{array}$ & $\begin{array}{l}\text { Right } \\
\text { Height }\end{array}$ & $\begin{array}{l}\text { Total } \\
\text { Height }\end{array}$ \\
\hline Visual acuity (logMAR) & $r=-0.57$ & $r=-0.53$ & $r=-0.05$ & $r=-0.43$ & $r=-0.21$ & $r=-0.22$ \\
& $p=0.013$ & $p=0.023$ & $p=0.84$ & $p=0.079$ & $p=0.41$ & $p=0.39$ \\
Optic nerve diameter & $r=0.4$ & $r=0.75$ & $r=-0.06$ & $r=-0.09$ & $r=0.09$ & $r=-0.03$ \\
& $p=0.047$ & $p<0.001$ & $p=0.78$ & $p=0.68$ & $p=0.66$ & $p=0.87$ \\
pRNFL thickness & $r=0.59$ & $r=0.54$ & $r=0.15$ & $r=0.38$ & $r=0.30$ & $r=0.28$ \\
& $p=0.003$ & $p=0.008$ & $p=0.49$ & $p=0.07$ & $p=0.16$ & $p=0.19$ \\
GCIPL volume & $r=0.55$ & $r=0.33$ & $r=0.19$ & $r=0.46$ & $r=0.34$ & $r=0.33$ \\
& $p=0.007$ & $p=0.13$ & $p=0.39$ & $p=0.028$ & $p=0.11$ & $p=0.12$ \\
\hline
\end{tabular}

$r$ values are Pearson's correlation coefficients. $N M O S D-O N=$ neuromyelitis optica patients with history of optic neuritis; $\log M A R=\operatorname{logarithm}$ of the minimum angle of resolution; $p R N F L=$ peripapillary retinal nerve fiber layer; $G C I P L=$ combined ganglion cell-inner plexiform layer 
might be equally limited in NMOSD and MS, since axonal loss is not the only substrate of neuronal atrophy and myelin loss, gliosis, and changes in water content also contribute to MRI-detected changes after ON [45]. Alongside findings suggesting that axonal loss is a major substrate of MRI-detected optic pathway atrophy after ON [17-20], the association between pRNFL (a surrogate for retinal axons) and OC measures implies that they are sensitive to atrophic changes of the anterior optic pathway. The association of OC area with visual function suggests a role for the $\mathrm{OC}$ as an imaging marker of neurodegenerative damage in the optic pathway with potential functional relevance.

Despite MRI's broad availability, no standardized MRI method for evaluation of optic pathway degeneration in standard scans is available. One major problem in optic nerve assessment is defining standardized measurement locations along the variable course of the nerve, which has high interindividual variability even in healthy populations $[3,4,15$, 17]. Several methods to measure optic nerve dimensions have been put forward [16, 17, 32]. These methods typically involve dedicated orbital MRI fat-saturated acquisition sequences along the axis of the optic nerve [3] additional to the commonly acquired sequences and extend the scan time for each patient. Others involve complex imaging postprocessing procedures [16] and, thus, may be difficult to implement in the routine clinical workup. Moreover, motion artifacts from eye movements and contrast reduction in the posterior region of the optic nerve, due to thinning of the CSF filled sub-arachnoid space, render MRI-based optic nerve assessment technically difficult [16]. This limits the accessibility of optic nerve measurements using MRI in the clinical setting.

The OC is less vulnerable to motion artifacts and consistently surrounded by CSF. It is less variable in morphology, bigger in dimensions and, thus, a simple target for MR investigations. OC assessment, as suggested in our study, only requires a broadly available MRI sequence (3D T1-weighted). While it does not provide information on the origin of the fibers and evaluation of focal optic nerve damage might better be achieved by direct measurement at the sight of inflammation, it accumulates neurodegeneration from both sides of the anterior optic pathway causing observable impairment. Thus, it extends the amount of information from a single measurement in the conventional and clinical standard scan, which can be used for monitoring of disease progression or therapeutic effectiveness.

Despite the low total number of subjects included in the study owing to the low prevalence of the disease, a significant difference in OC measures was shown within a relatively large homogeneous cohort exclusively consisting of AQP4-IgGpositive NMOSD patients. Separate gender analysis could not be conducted due to the high proportion of female patients. This is in line with the strong female preponderance in NMOSD [46]. OC width was measured along straight lines, which may not account for curved width. In future investigations, curved lines could be drawn; however, only few participants showed recognizable deviation from straight lines and, thus, we do not expect that this would drastically change the presented results.

Our data provide a strong rationale for future, larger studies on OC measures in ON, including in NMOSD patients with acute $\mathrm{ON}$, in which inflammation might result in an increase in OC dimensions, as well as in patients with inflammatory diseases such as MS and myelin oligodendrocyte glycoprotein antibody associated disease (MOGAD) [47]. Finally, studies on the influence of susceptibility artifacts on scanners with different field strength and resolution seem justified and the application of advanced quantitative imaging methods such as DTI could reveal insights into the relationship between anterior and posterior optic pathway neurodegeneration.

\section{Conclusion}

Our study represents an initial and thorough assessment of OC measures to evaluate optic pathway degeneration using standard MRI and shows that the OC area is suitable and reliable. This simple method extends the amount of information that can be obtained from conventional and clinically available scans. Our results suggest that the $\mathrm{OC}$ might evolve into an easily accessible imaging marker of neurodegeneration in the anterior optic pathway with potential functional relevance.

Acknowledgments MR imaging for this study was performed at the Berlin Center for Advanced Neuroimaging. The authors thank the participants of the study, Susan Pikol, Cynthia Kraut, and Charlotte Bereuter for their excellent technical support.

Funding information Open Access funding provided by Projekt DEAL. The authors state that this work has not received any funding.

\section{Compliance with ethical standards}

Guarantor The scientific guarantor of this publication is Friedemann Paul.

Conflict of interest The authors of this manuscript declare relationships with the following companies:

NS has received travel grants from Biogen Idec and sanofi-aventis/ Genzyme.

SA received travel grants from Celgene $\mathrm{GmbH}$, unrelated to this project.

FCO was employed by Nocturne, unrelated to this project.

$\mathrm{HZ}$ received research grants from Novartis, unrelated to this project.

KR was supported by the German Ministry of Education and Research (BMBF/KKNMS, Competence Network Multiple Sclerosis) and has received research support from Novartis and Merck Serono as well as speaking fees and travel grants from Guthy Jackson Charitable Foundation, Bayer Healthcare, Biogen Idec, Merck Serono, sanofiaventis/Genzyme, Teva Pharmaceuticals, Roche, and Novartis. 
JBS has received travel grants and speaking fees from Bayer Healthcare, Biogen Idec, Merck Serono, sanofi-aventis/Genzyme, Teva Pharmaceuticals, and Novartis.

FP declares that he has received research grants and speaker's honoraria from Bayer Healthcare, Teva Pharmaceuticals, Genzyme, Merck and Co., Novartis, and MedImmune. He is also a member of the steering committee for the OCTIMS study (run by Novartis).

AUB is cofounder and shareholder of technology start-ups Motognosis and Nocturne. He is named as inventor on several patent applications describing MS serum biomarkers, perceptive visual computing for motor function assessment and retinal image analysis.

Statistics and biometry No complex statistical methods were necessary for this paper.

Informed consent Written informed consent was obtained from all subjects (patients) in this study.

Ethical approval Institutional Review Board approval was obtained.

Study subjects or cohorts overlap Some study subjects or cohorts have been previously reported in PMID 31127016.

\section{Methodology}

- Retrospective

- Observational

- Performed at one institution

Open Access This article is licensed under a Creative Commons Attribution 4.0 International License, which permits use, sharing, adaptation, distribution and reproduction in any medium or format, as long as you give appropriate credit to the original author(s) and the source, provide a link to the Creative Commons licence, and indicate if changes were made. The images or other third party material in this article are included in the article's Creative Commons licence, unless indicated otherwise in a credit line to the material. If material is not included in the article's Creative Commons licence and your intended use is not permitted by statutory regulation or exceeds the permitted use, you will need to obtain permission directly from the copyright holder. To view a copy of this licence, visit http://creativecommons.org/licenses/by/4.0/.

\section{References}

1. Jarius S, Wildemann B, Paul F (2014) Neuromyelitis optica: clinical features, immunopathogenesis and treatment. Clin Exp Immunol 176(2):149-164

2. Waters P, Reindl M, Saiz A et al (2016) Multicentre comparison of a diagnostic assay: aquaporin-4 antibodies in neuromyelitis optica. $\mathrm{J}$ Neurol Neurosurg Psychiatry 87(9):1005-1015

3. Hickman SJ, Brex PA, Brierley CM et al (2001) Detection of optic nerve atrophy following a single episode of unilateral optic neuritis by MRI using a fat-saturated short-echo fast FLAIR sequence. Neuroradiology 43:123-128

4. Hickman SJ, Toosy AT, Jones SJ et al (2004) A serial MRI study following optic nerve mean area in acute optic neuritis. Brain 127: 2498-2505

5. Tian DC, Su L, Fan M et al (2018) Bidirectional degeneration in the visual pathway in neuromyelitis optica spectrum disorder (NMOSD). Mult Scler 24:1585-1593
6. Tur C, Goodkin O, Altmann DR et al (2016) Longitudinal evidence for anterograde trans-synaptic degeneration after optic neuritis. Brain 139:816-828

7. Gabilondo I, Martinez-Lapiscina EH, Martinez-Heras E et al (2014) Trans-synaptic axonal degeneration in the visual pathway in multiple sclerosis. Ann Neurol 75:98-107

8. Sinnecker T, Oberwahrenbrock T, Metz I et al (2015) Optic radiation damage in multiple sclerosis is associated with visual dysfunction and retinal thinning an ultrahigh-field MR pilot study. Eur Radiol 25:122-131

9. Kuchling J, Backner Y, Oertel FC et al (2018) Comparison of probabilistic tractography and tract-based spatial statistics for assessing optic radiation damage in patients with autoimmune inflammatory disorders of the central nervous system. Neuroimage Clin 19:538550

10. Kuchling J, Brandt AU, Paul F, Scheel M (2017) Diffusion tensor imaging for multilevel assessment of the visual pathway: possibilities for personalized outcome prediction in autoimmune disorders of the central nervous system. EPMA J 8:279-294

11. Merle H, Olindo S, Bonnan M et al (2007) Natural history of the visual impairment of relapsing neuromyelitis optica. Ophthalmology 114:810-815

12. Schmidt F, Zimmermann H, Mikolajczak J et al (2017) Severe structural and functional visual system damage leads to profound loss of vision-related quality of life in patients with neuromyelitis optica spectrum disorders. Mult Scler Relat Disord 11:45-50

13. Stiebel-Kalish H, Hellmann MA, Mimouni M et al (2019) Does time equal vision in the acute treatment of a cohort of AQP4 and MOG optic neuritis? Neurol Neuroimmunol Neuroinflamm 6:e572

14. Beekman J, Keisler A, Pedraza O et al (2019) Neuromyelitis optica spectrum disorder: patient experience and quality of life. Neurol Neuroimmunol Neuroinflamm 6:e580

15. Hickman SJ, Brierley CM, Brex PA et al (2002) Continuing optic nerve atrophy following optic neuritis: a serial MRI study. Mult Scler 8:339-342

16. Harrigan RL, Smith AK, Lyttle B et al (2017) Quantitative characterization of optic nerve atrophy in patients with multiple sclerosis. Mult Scler J Exp Transl Clin 3:2055217317730097

17. Trip SA, Schlottmann PG, Jones SJ et al (2006) Optic nerve atrophy and retinal nerve fibre layer thinning following optic neuritis: evidence that axonal loss is a substrate of MRI-detected atrophy. Neuroimage. 31:286-293

18. Parravano JG, Toledo A, Kucharczyk W (1993) Dimensions of the optic nerves, chiasm, and tracts: MR quantitative comparison between patients with optic atrophy and normals. J Comput Assist Tomogr 17:688-690

19. Manogaran P, Vavasour IM, Lange AP et al (2016) Quantifying visual pathway axonal and myelin loss in multiple sclerosis and neuromyelitis optica. Neuroimage Clin 11:743-750

20. Manogaran P, Hanson JV, Olbert ED et al (2016) Optical coherence tomography and magnetic resonance imaging in multiple sclerosis and Neuromyelitis Optica Spectrum disorder. Int J Mol Sci 17: e1894

21. Trip SA, Schlottmann PG, Jones SJ et al (2005) Retinal nerve fiber layer axonal loss and visual dysfunction in optic neuritis. Ann Neurol 58:383-391

22. Oertel FC, Zimmermann HG, Brandt AU, Paul F (2018) Novel uses of retinal imaging with optical coherence tomography in multiple sclerosis. Expert Rev Neurother 19:31-43

23. Oberwahrenbrock T, Traber GL, Lukas S et al (2018) Multicenter reliability of semiautomatic retinal layer segmentation using OCT. Neurol Neuroimmunol Neuroinflamm 5:e449

24. Dorr J, Wernecke KD, Bock M et al (2011) Association of retinal and macular damage with brain atrophy in multiple sclerosis. PLoS One 6:e18132 
25. Ayadi N, Dörr J, Motamedi S (2018) Temporal visual resolution and disease severity in MS. Neurol Neuroimmunol Neuroinflamm 5(5): 49

26. Oertel FC, Zimmermann H, Paul F, Brandt AU (2018) Optical coherence tomography in neuromyelitis optica spectrum disorders: potential advantages for individualized monitoring of progression and therapy. EPMA J 9:21-33

27. Oertel FC, Kuchling J, Zimmermann H et al (2017) Microstructural visual system changes in AQP4-antibody-seropositive NMOSD. Neurol Neuroimmunol Neuroinflamm 4:e334

28. Bennett JL, de Seze J, Lana-Peixoto M et al (2015) Neuromyelitis optica and multiple sclerosis: seeing differences through optical coherence tomography. Mult Scler 21:678-688

29. Wingerchuk DM, Banwell B, Bennett JL et al (2015) International consensus diagnostic criteria for neuromyelitis optica spectrum disorders. Neurology 85:177-189

30. Kim HJ, Paul F, Lana-Peixoto MA et al (2015) MRI characteristics of neuromyelitis optica spectrum disorder: an international update. Neurology 84:1165-1173

31. Wagner AL, Murtagh FR, Hazlett KS, Arrington JA (1997) Measurement of the normal optic chiasm on coronal MR images. AJNR Am J Neuroradiol 18:723-726

32. Avery RA, Mansoor A, Idrees R et al (2016) Quantitative MRI criteria for optic pathway enlargement in neurofibromatosis type 1. Neurology 86:2264-2270

33. Kasmann-Kellner B, Schafer T, Krick CM, Ruprecht KW, Reith W, Schmitz BL (2003) Anatomical differences in optic nerve, chiasma and tractus opticus in human albinism as demonstrated by standardized clinical and MRI evaluation. Klin Monbl Augenheilkd 220: 334-344

34. Kurtzke JF (1983) Rating neurologic impairment in multiple sclerosis. Neurology. 33:1444-1452

35. Cruz-Herranz A, Balk LJ, Oberwahrenbrock T et al (2016) The APOSTEL recommendations for reporting quantitative optical coherence tomography studies. Neurology 86:2303-2309

36. Tewarie P, Balk L, Costello F et al (2012) The OSCAR-IB consensus criteria for retinal OCT quality assessment. PLoS One 7:e34823
37. Schippling S, Balk L, Costello F et al (2015) Quality control for retinal OCT in multiple sclerosis: validation of the OSCAR-IB criteria. Mult Scler 21:163-170

38. Hajian-Tilaki K (2013) Receiver operating characteristic (ROC) curve analysis for medical diagnostic test evaluation. Caspian $\mathrm{J}$ Intern Med 4:627-635

39. Smith SM, ThangY JM et al (2002) Accurate, robust, and automated longitudinal and cross-sectional brain change analysis. Neuroimage 17:479-489

40. Wickham H (2017) Tidyverse: easily Install and Load the “Tidyverse.". Available via https://cranr-project.org/package. Accessed 10 Oct 2018

41. Kassambara A (2017) ggpubr: "ggplot2" Based Publication Ready Plots. Available via https://cran.r-project.org/web/packages/ggpubr/ index.html. Accessed 10 Oct 102018

42. Robin X, Turck N, Hainard A et al (2011) pROC: an open-source package for $\mathrm{R}$ and $\mathrm{S}+$ to analyze and compare ROC curves. BMC Bioinformatics 12:77

43. Akaishi T, Kaneko K, Himori N et al (2017) Subclinical retinal atrophy in the unaffected fellow eyes of multiple sclerosis and neuromyelitis optica. J Neuroimmunol 313:10-15

44. Oertel FC, Havla J, Roca-Fernandez A et al (2018) Retinal ganglion cell loss in neuromyelitis optica: a longitudinal study. J Neurol Neurosurg Psychiatry 89:1259-1265

45. Noval S, Contreras I, Muñoz S, Oreja-Guevara C, Manzano B, Rebolleda G (2011) Optical coherence tomography in multiple sclerosis and neuromyelitis optica: an update. Mult Scler Int 2011: 472790

46. Gold SM, Willing A, Leypoldt F, Paul F, Friese MA (2019) Sex differences in autoimmune disorders of the central nervous system. Semin Immunopathol 41:177-188

47. Borisow N, Mori M, Kuwabara S, Scheel M, Paul F (2018) Diagnosis and treatment of NMO spectrum disorder and MOGencephalomyelitis. Front Neurol 9:888

Publisher's note Springer Nature remains neutral with regard to jurisdictional claims in published maps and institutional affiliations. 